\title{
Lomnitz-type viscoelastic behavior of clear spruce wood as identified by creep and relaxation experiments: influence of moisture content and elevated temperatures up to $80^{\circ} \mathrm{C}$
}

\author{
U. Hofer ${ }^{1}$ (D) C. Pichler ${ }^{1} \cdot$ R. Maderebner ${ }^{2} \cdot$ R. Lackner ${ }^{1}$
}

Received: 17 January 2019 / Published online: 18 May 2019

(c) The Author(s) 2019

\begin{abstract}
The strong dependence of viscoelastic behavior of wood on temperature and moisture content is well documented in the literature. In this paper, viscoelastic behavior of spruce wood is monitored experimentally and modeled with a (logarithmic) Lomnitz-type creep model. Creep tests are performed at different levels of temperature $\left(25-80^{\circ} \mathrm{C}\right)$ and moisture content $(0$ to $\approx 15$ mass- $\%)$. The variation of Lomnitz parameters as regards temperature and moisture content is well captured by Arrhenius-type dependencies. Furthermore, the uniqueness of the results is discussed and relaxation tests are performed in order to verify the so-obtained viscoelastic parameters.
\end{abstract}

\section{Introduction}

Wood may be idealized as a fiber-reinforced polymer, with the microfibrils of cellulose acting as fibers and reinforcing the wood in longitudinal direction, and the polymeric hemicellulose and lignin acting as a matrix material embedding the cellulose fibers (Holzer et al. 1989; Hofstetter et al. 2005; Bader et al. 2011). This matrix additionally contains distinct porous structures. As many other polymers, woodespecially the amorphous phases hemicellulose and lignin (Kelley et al. 1987) shows a pronounced time-dependent behavior (Navi and Stanzl-Tschegg 2009). In this regard, one needs to distinguish between transport-induced, load-induced, and pseudo (shrinkage and swelling) viscoelastic phenomena, respectively (Hunt 1999).

U. Hofer

Ulrich.Hofer@uibk.ac.at

1 Material Technology Innsbruck (MTI), University of Innsbruck, Technikerstraße 13, 6020 Innsbruck, Austria

2 Unit for Timber Engineering, University of Innsbruck, Technikerstraße 13, 6020 Innsbruck, Austria 
It is well documented that relative humidity changes during a creep test accelerate creep, disregarding whether humidity increases or decreases (Armstrong and Kingston 1960; Ranta-Maunus 1975; Bažant and Meiri 1985; Mukudai and Yata 1986; Mohager and Toratti 1992). This phenomenon is commonly denoted as mechano-sorptive creep. Bažant (1985) identified mechano-sorptive creep as a transportinduced phenomenon taking place in the porous structure of wood, roughly idealized as a network consisting of micro- and macropores. On the one hand, macropores are responsible for water transport throughout the meso-structure of wood. At least for moderate loading rates, water contained in those pores cannot withstand any external stresses. On the other hand, structured water molecules within micropores can act against the external stresses, and, hence, can transmit external loads, c.f. Bažant (1985). Although those micropores are not involved in any global water transport, however, water molecules are transported within this microporous structures in the scope of local diffusion processes caused by moisture gradients. This molecular transport causes breakage and reforming of hydrogen bonds in the wood microfibrils, which is identified as the source of mechano-sorptive creep in wood. It is important to note that in the case of constant overall moisture content, no water transport through the macropores and no diffusion processes within micropores, respectively, occur. Hence, mechano-sorptive creep is deactivated. A similar effect is observed for cement-based materials, the so-called Pickett-effect (Pickett 1942; Acker and Ulm 2001), which is explained on the one hand by microcracking and on the other hand by a gradient in the moisture content causing molecular water transport within gel pores and hence breakage and reformation of $\mathrm{H}$-bonds (similar to creep in wood), c.f. Bažant and Xi (1993) and Altoubat and Lange (2002).

As a second source of time-dependent behavior, load-induced (or viscoelastic) creep is observed in wood, occurring at constant moisture content within the wood. The molecular mechanism of viscoelastic creep, the breaking and reformation of H-bonds, is similar to mechano-sorptive creep (Bodig and Jayne 1982). An increased moisture content leads to augmented H-bond breakage and therefore to increased creep rates (Hering and Niemz 2012); similarly, higher temperatures increase the creep rate of wood (Jiang et al. 2009), probably due to the higher thermal motion of water molecules and therefore again due to an increase in hydrogen bond breakage. Due to the longitudinal alignment of cellulose fibers, the elastic and viscoelastic behavior of wood is apparently strongly anisotropic, which was investigated by Schniewind and Barrett (1972) and Ozyhar et al. (2013), where the latter authors distinguished between tension and compression.

Pseudo-creep (shrinkage and swelling), in contrast to mechano-sorptive and viscoelastic creep, is a fully reversible process (Hunt 1999; Hunt and Shelton 1988). The underlying physical process for swelling (shrinkage) is the absorption (desorption) of water in the cell walls (Nakano 2008). Shrinkage and swelling take place at moisture contents between oven-dry conditions and the fiber-saturation point and its magnitude is a function of the moisture content (Stamm 1935). Additionally, the microstructure plays a fundamental role in the amount of shrinkage and swelling (Schroeder 1972).

As regards models for describing viscoelastic behavior, several classical models were compared by Haque et al. (2000), finding that the Kelvin-Voigt model 
and the Burgers model are best suited to describe creep of Pinus radiata at different levels of moisture content. Hering and Niemz (2012) used a Kelvin-Voigt model to describe the moisture content-dependent creep of beech wood. Mukudai (1983) used a Maxwell model and three Kelvin-Voigt models connected in series to model the viscoelastic behavior of Japanese cypress, whereas Hunt (2004) used a generalized Kelvin-Voigt model to estimate the creep curves of wood. Clouser (1959), Schniewind and Barrett (1972) and Hoyle et al. (1985) employed power-law models to model creep behavior of timber beams. Eitelberger et al. (2012) modeled the viscoelastic behavior of the lignin-hemicellulose matrix with a power-law model, whereas the macroscopic wood behavior was predicted to follow a fractional Zener model. King (1961) observed that the creep deformation of wood is proportional to time and hence introduced a logarithmic creep model to consider the viscoelastic behavior of wood, whereas Bach and Pentoney (1968) introduced a modified logarithmic creep law. In geology, a logarithmic creep model, the so-called Lomnitz model, has been introduced by Lomnitz (1956, 1957, 1962). So far it has been applied to model cement-based materials (e.g., in Bažant and Prasannan 1989; Bažant et al. 1997; Acker and Ulm 2001; Pichler et al. 2008; Pichler and Lackner 2009), polyurethane foams (Pichler et al. 2018) and metals (Nabarro 2001a, b).

For wood, on the other hand, logarithmic models have been introduced by King (1961) and Bach and Pentoney (1968), but have not been applied to the thermohygro-mechanical analysis of wood and wood structures. That may be (i) due to the missing expression for the relaxation function, which cannot be obtained in a straightforward manner and has only been derived recently by Mainardi and Spada (2012), and (ii) due to the lack of a physical interpretation of logarithmic creep laws, which has been given only recently by Pandey and Holm (2016) by linking timedependent Newtonian viscosity to the parameters of the Lomnitz model. With these recent developments, the Lomnitz model has become much more attractive, also on account of the smaller number of model parameters, as compared to other models (e.g., the generalized Kelvin-Voigt or the generalized Maxwell model), necessary for representation of test data.

Therefore, the present paper readopts the logarithmic Lomnitz model for wood and considers, in addition to King (1961) and Bach and Pentoney (1968), the influence of temperature (Morlier 1994; Bekhta and Niemz 2003) and moisture content (Schänzlin 2010), as those parameters are commonly known to majorly influence the time-dependent behavior. For this purpose, the viscoelastic behavior of clear (knotfree) wood specimens of spruce at the $10 \mathrm{~mm}$ scale of observation is determined, disabling mechano-sorptive creep as well as shrinkage and swelling. Half-hour long creep tests were performed at different (constant) levels of temperature and moisture content.

The paper is structured as follows: in "The viscoelastic Lomnitz law" section, the logarithmic Lomnitz law is briefly reviewed. In the "Experimental work" section, the experimental methods are described, which give access to data employed for identification of the viscoelastic parameters of the Lomnitz law as described in the "Parameter identification" section. The uniqueness of the so-obtained parameters is discussed in the "Uniqueness analysis of fitted material parameters" section, while the results are 
discussed in the "Discussion" section. The paper closes with final remarks in the "Conclusion" section.

\section{Materials and methods}

\section{The viscoelastic Lomnitz law}

The Lomnitz-type creep law, first introduced by Lomnitz (1956), assumes that the strain rate in a creep test is inversely proportional to time, i.e., $\mathrm{d} \varepsilon / \mathrm{d} t \propto 1 / t$. The uniaxial creep compliance $J$, defined as $\varepsilon(t) / \sigma$, for a constant stress applied at $t=0$, i.e., $\sigma(t)=H(t) \sigma_{0}$, reads

$$
J=\frac{1}{E}+J_{\log } \ln \left(1+t / \tau_{\log }\right)
$$

with $E$ as the materials Young's modulus, $J_{\log }$ as a creep parameter and $\tau_{\log }$ as the characteristic time of the creep process. The creep compliance $J$ can be split into an instantaneous part $J_{0}$ and a time-dependent part $J_{v}(t)$ :

$$
J=J_{0}+J_{v}(t)
$$

where $J_{0}$ is the inverse of the Young's modulus, i.e., $J_{0}=1 / E$, and $J_{v}(t)$ can be expressed in terms of time-dependent bulk and shear moduli, respectively (Brinson and Brinson 2008):

$$
J_{v}(t)=\frac{3 K(t)+G(t)}{9 K(t) G(t)}
$$

Inserting Eq. (3) into Eq. (2) yields

$$
J=J_{0}+\frac{3 K(t)+G(t)}{9 K(t) G(t)}=\frac{1}{E}+\frac{1}{9} \frac{1}{K(t)}+\frac{1}{3} \frac{1}{G(t)}=\frac{1}{E}+\frac{1}{9} J^{\mathrm{vol}}+\frac{1}{3} J^{\mathrm{dev}}
$$

with $J^{\mathrm{vol}}=1 / K(t)$ and $J^{\mathrm{dev}}=1 / G(t)$ denoting the volumetric and deviatoric creep compliances, respectively. As viscoelastic deformations in polymers are assumed to be caused by a sliding mechanism at the microscale (bond breakage in shear directions), viscoelasticity is assumed to be purely deviatoric (Ward and Sweeney 2012). This assumption is commonly adapted, see for example, Brinson and Brinson (2008), Pichler et al. (2018), Hofer et al. (2018), Beijer and Spoormaker (2002), Idesman et al. (2001) and Singh and Rosenman (1974). As hemicellulose and lignin may be characterized as natural polymers, this assumption is also presupposed in the present paper, hence, the volumetric compliance $J^{\mathrm{vol}}$ is set to zero. Considering the Lomnitz model [Eq. (1)] for the deviatoric compliance in Eq. (4) results in the deviatoric Lomnitz model (Fig. 1a) as

$$
J=\frac{1}{E}+\frac{1}{3} J_{\log }^{\mathrm{dev}} \ln \left(1+t / \tau_{\log }\right)
$$

with $J_{\log }^{\mathrm{dev}}$ as the deviatoric creep compliance parameter. 
(a)

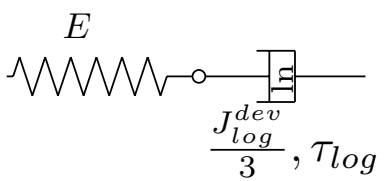

(b)

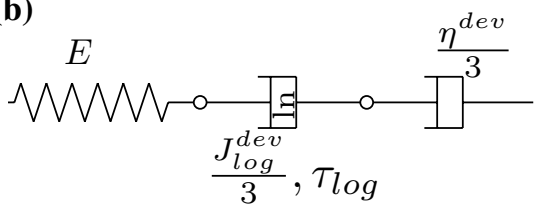

Fig. 1 Uniaxial representation of Lomnitz model (a) and extended Lomnitz model (b)

Pichler et al. (2018) considered a finite loading $\operatorname{ramp}$ from $t=0$ to $t=t_{0}$ as $\sigma(t)=\sigma_{0} t / t_{0}$ for $0 \leq t \leq t_{0}$ giving the so-called ramp compliance $\widetilde{J}$ as

$$
\widetilde{J}\left(t>t_{0}\right)=\frac{\varepsilon(t)}{\sigma_{0}}=\frac{1}{E}+\frac{1}{3} J_{\log }^{\operatorname{dev}}\left[\frac{t+\tau_{\log }}{t_{0}} \ln \left(1+\frac{t}{\tau_{\log }}\right)-\frac{t-t_{0}+\tau_{\log }}{t_{0}} \ln \left(1+\frac{t-t_{0}}{\tau_{\log }}\right)-1\right]
$$

In order to improve the versatility of the model (i.e., improve the quality of fit of model to the test data, c.f. "Uniqueness analysis of fitted material parameters" section), the Lomnitz model is extended in series by a Newtonian dashpot (Flügge 1975) (referred to as extended Lomnitz model in the remainder of this paper), resulting in

$$
J=\frac{1}{E}+\frac{1}{3} J_{\log }^{\operatorname{dev}} \ln \left(1+t / \tau_{\log }\right)+\frac{1}{3} \frac{t}{\eta^{\operatorname{dev}}}
$$

with $\eta^{\text {dev }}$ as the (deviatoric) viscosity of the dashpot (see Fig. 1b). In terms of the ramp compliance, Eq. (7) becomes

$$
\widetilde{J}\left(t>t_{0}\right)=\frac{1}{E}+\frac{1}{3} J_{\log }^{\mathrm{dev}}\left[\frac{t+\tau_{\log }}{t_{0}} \ln \left(1+\frac{t}{\tau_{\log }}\right)-\frac{t-t_{0}+\tau_{\log }}{t_{0}} \ln \left(1+\frac{t-t_{0}}{\tau_{\log }}\right)-1\right]+\frac{1}{3} \frac{t}{\eta^{\operatorname{dev}}}
$$

The derivatives of Eqs. (6) and (8) are given as

$$
\dot{\widetilde{J}}\left(t>t_{0}\right)=\frac{1}{3} \frac{J_{\log }^{\mathrm{dev}}}{t_{0}} \ln \left(\frac{t+\tau_{\log }}{t-t_{0}+\tau_{\log }}\right)
$$

and

$$
\dot{\widetilde{J}}\left(t>t_{0}\right)=\frac{1}{3} \frac{J_{\log }^{\mathrm{dev}}}{t_{0}} \ln \left(\frac{t+\tau_{\log }}{t-t_{0}+\tau_{\log }}\right)+\frac{1}{3} \frac{1}{\eta^{\mathrm{dev}}},
$$

respectively. The relaxation modulus $R(t)$ is connected to the creep compliance $J(t)$ via their respective Laplace-Carson transformations using the reciprocity principle:

$$
\mathscr{L} \mathscr{C}[R(t)]=\frac{1}{\mathscr{L} \mathscr{C}[J(t)]} \quad \Leftrightarrow \quad R^{*}(p)=\frac{1}{J^{*}(p)}
$$

The Laplace-Carson transformation of Eq. (7) results in 


$$
J^{*}(p)=\frac{1}{E}+\frac{1}{3} J_{\log }^{\mathrm{dev}} \exp \left(p \tau_{\log }\right) \Gamma\left(0, p \tau_{\log }\right)+\frac{1}{p} \frac{1}{3 \eta^{\mathrm{dev}}}
$$

with $\Gamma(a, z)=\int_{z}^{\infty} t^{a-1} \exp (-t) \mathrm{d} t$ as the incomplete gamma function. Since the inverse of $J^{*}(p)$ cannot be transformed into time domain by analytical means, no closed-form expression can be obtained for the relaxation modulus by exploiting the reciprocity principle. However, Mainardi and Spada (2012) derived an analytical expression for the relaxation modulus by relating the relaxation and compliance function by a Volterra integral equation (Pipkin 1986):

$$
R(t)=\frac{1}{J(t=0)}-\frac{1}{J(t=0)} \int_{0}^{t} \dot{J}\left(t-t^{\prime}\right) R\left(t^{\prime}\right) \mathrm{d} t^{\prime}
$$

By using Eq. (5) and expressing the relaxation modulus as $R(t)=\phi(t) E$, Eq. (13) becomes

$$
R(t)=\phi(t) E=\left[1+\int_{0}^{t} \kappa\left(t, t^{\prime}\right) \phi\left(t^{\prime}\right) \mathrm{d} t^{\prime}\right] E
$$

with the solution of the associated Volterra equation

$$
\phi(t)=1+\int_{0}^{t} \kappa\left(t, t^{\prime}\right) \phi\left(t^{\prime}\right) \mathrm{d} t^{\prime}
$$

obtained numerically employing (Press et al. 1992), and the (creep) kernel defined as

$$
\kappa\left(t, t^{\prime}\right)=-\frac{E}{3}\left(\frac{J_{\log }^{\mathrm{dev}}}{t-t^{\prime}+\tau_{\log }}+\frac{1}{\eta^{\mathrm{dev}}}\right)
$$

\section{Experimental work}

Clear wood specimens of spruce (Picea abies), with the dimensions $B \times H \times L=20 \times 20 \times 280 \mathrm{~mm}$, are cut such that the grain direction and the annual rings are parallel to the surface, see Fig. 2.

The spruce specimens were conditioned at four different temperatures, $T=25^{\circ} \mathrm{C}$, $T=40^{\circ} \mathrm{C}, T=65^{\circ} \mathrm{C}, T=80^{\circ} \mathrm{C}$, and at different relative humidity conditions. The varying relative humidities were controlled either by subjecting specimens to an atmosphere associated with saturated salt solutions or direct conditioning in climate chambers. Oven-dried specimens (line \#1 in Table 1) were dried at $T=105^{\circ} \mathrm{C}$ in an oven before exposing them to the specified testing temperature. Note that ovendrying has only a negligible influence on the viscoelastic properties of dry wood, i.e., the microstructure of the wood is not altered by oven-drying (Stamm 1956; Placet et al. 2008; Bekhta and Niemz 2003). Specimens referring to lines \#2-\#5 in Table 1 were conditioned in glass boxes containing saturated salt solutions (see Table 2, Greenspan 1977); those boxes were then placed in climate chambers with 


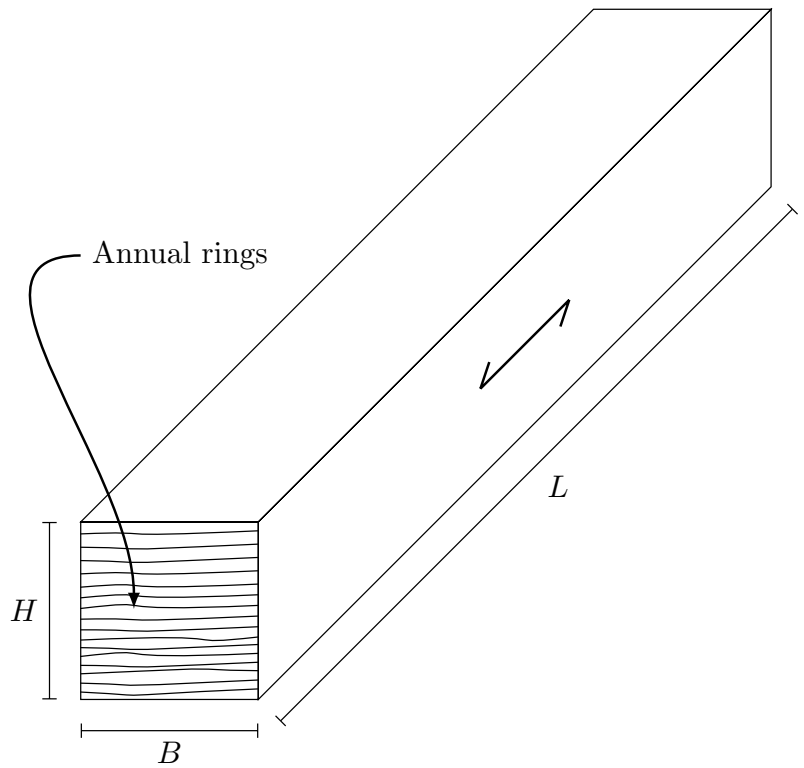

Fig. 2 Spruce specimen: dimensions and grain direction

Table 1 Wood moisture contents (mass-\%) obtained by conditioning the specimens at different temperature levels, average values of 6 specimens

\begin{tabular}{lllll}
\hline & $25^{\circ} \mathrm{C}$ & $40{ }^{\circ} \mathrm{C}$ & $65^{\circ} \mathrm{C}$ & $80{ }^{\circ} \mathrm{C}$ \\
\hline$\# 1$ & Oven-dry & Oven-dry & Oven-dry & Oven-dry \\
$\# 2$ & 7.1 & 5.7 & 4.6 & 3.2 \\
$\# 3$ & 9.6 & 8.2 & 6.6 & 4.0 \\
$\# 4$ & 12.9 & 10.3 & 8.2 & 6.7 \\
$\# 5$ & 15.6 & 14.6 & 14.1 & 8.3 \\
$\# 6$ & - & - & - & 12.8 \\
\hline
\end{tabular}

Table 2 Relative humidity (\%) in atmosphere above saturated salt solutions according to Greenspan (1977), giving $4 \times 4=16$ conditioning classes

\begin{tabular}{lllll}
\hline$T\left({ }^{\circ} \mathrm{C}\right)$ & Lithium chloride $(\mathrm{LiCl})$ & $\begin{array}{l}\text { Magnesium } \\
\text { chloride } \\
\left(\mathrm{MgCl}_{2}\right)\end{array}$ & Sodium bromide $(\mathrm{NaBr})$ & Sodium chloride $(\mathrm{NaCl})$ \\
\hline 25 & $11.30 \pm 0.27$ & $32.78 \pm 0.16$ & $57.57 \pm 0.40$ & $75.29 \pm 0.12$ \\
40 & $11.21 \pm 0.21$ & $31.60 \pm 0.13$ & $53.17 \pm 0.41$ & $74.68 \pm 0.13$ \\
65 & $10.86 \pm 0.29$ & $28.54 \pm 0.18$ & $49.49 \pm 0.94$ & $74.71 \pm 0.37$ \\
80 & $10.51 \pm 0.44$ & $26.05 \pm 0.34$ & $51.43 \pm 1.50$ & $76.29 \pm 0.65$ \\
\hline
\end{tabular}

temperatures of $T=25^{\circ} \mathrm{C}, T=40^{\circ} \mathrm{C}, T=65^{\circ} \mathrm{C}, T=80^{\circ} \mathrm{C}$, respectively. Specimens referring to line \#6 of Table 1 were conditioned in a climate chamber at 
$T=80^{\circ} \mathrm{C}$ and relative air humidity of $90 \%$. The specimens were conditioned until their weight changed less than $1 \%$ in two consecutive weighings (separated by more than one day).

In total, 21 conditioning classes were employed, leading to average wood moisture contents as shown in Table 1. The specimens were assigned to the conditioning classes such that similar densities are evenly distributed among all classes. For each class, six specimens were conditioned, leading to a total amount of 126 specimens employed in 126 creep tests.

Three-point-bending creep tests with load application at half-span and length between the two supports of $L_{s}=240 \mathrm{~mm}$ were employed to determine the viscoelastic behavior. A Shimadzu AG-X plus $10 \mathrm{kN}$ testing frame was used for all tests, exhibiting a characteristic tolerance of $\pm 0.3 \%$ as regards the applied force. A constant load $F=555 \mathrm{~N}$, corresponding to an edge normal stress of about $25 \mathrm{MPa}$ [far below the flexural strength of $\approx 95 \mathrm{MPa}$ (Austrian Standards International 2003)], was applied with a loading rate of $\dot{F}=20 \mathrm{~N} / \mathrm{s}$; the load was kept constant for $1800 \mathrm{~s}$. The mid-span vertical displacement $u(t)$, representing the bending deflection of the beam and measured in the direction of the applied load, was monitored with an inductive displacement transducer (HBM WA20) with a characteristic tolerance of $\pm 1 \%$. The data acquisition rate was set to $5 \mathrm{~Hz}$ throughout the experiments. After the $1800 \mathrm{~s}$ creep phase, the specimen was unloaded, whereupon small deformations remained. Each test was conducted at the temperature which has been prescribed previously during conditioning, i.e., the temperature given in Table 1. As an exception, the oven-dried specimens were conditioned at $T=105^{\circ} \mathrm{C}$ but tested at lower temperatures $\left(T=25^{\circ} \mathrm{C}, T=40^{\circ} \mathrm{C}, T=65^{\circ} \mathrm{C}\right.$ and $\left.T=80^{\circ} \mathrm{C}\right)$. The transport of specimens from the conditioning domain, i.e., salt box or climate chamber, to the thermostatic chamber of the testing frame was done within less than a minute, hence the temperature remained almost constant during the transition from conditioning to testing. With the Shimadzu thermostatic chamber TCE-N300, the temperature was kept constant during testing, which was verified at start and end of the tests with a temperature sensor (Testo $177-\mathrm{T} 4$, characteristic tolerance $\pm 0.3^{\circ} \mathrm{C}$ ) directly through a hole in the specimen (outside of the supports). The spruce specimens were wrapped in diffusion-resistant film to prevent sample drying during the half-hour creep test, which was verified by weighing the specimen immediately before and after the tests. By keeping the moisture content constant throughout the test, it was ensured that mechano-sorptive creep processes were not active. After performing a test, the specimen was oven-dried at $105^{\circ} \mathrm{C}$, hence the moisture content $w$ during testing can be obtained as

$$
w=\frac{m_{w e t}-m_{d r y}}{m_{d r y}}
$$

with $m_{w e t}$ as the mass of the wet specimen immediately after the test procedure and $m_{d r y}$ as the mass after oven-drying.

In addition to the aforementioned creep tests, four three-point bending relaxation tests were performed at temperature levels of $T=25^{\circ} \mathrm{C}, T=40^{\circ} \mathrm{C}, T=65^{\circ} \mathrm{C}$, and $T=80^{\circ} \mathrm{C}$, respectively, serving as independent verification experiments as regards 
material parameters back-calculated in the "Discussion" section. Test setup, equipment and testing procedure were exactly the same as for the creep tests, except that the specimens were subjected to a constant displacement $u_{0}=1.21 \mathrm{~mm}$ at midspan and the force history was recorded. The displacement was applied at a rate of $0.2 \mathrm{~mm} / \mathrm{s}$ (hence the duration for the load application was $\approx 6 \mathrm{~s}$ ) and kept constant for $1800 \mathrm{~s}$.

\section{Parameter identification}

The experimentally obtained displacement histories are used to calculate the creep compliance $J_{\text {test }}$ as

$$
J_{\text {test }}(t)=4 \frac{B H^{3}}{F L_{s}^{3}} u(t)
$$

where linear viscoelastic behavior is assumed and the specimen is idealized as an Euler-Bernoulli beam, i.e., the beam is assumed to be slender (ratio $L / H=12$ ), hence, shear deformation can be neglected and the displacements are small, i.e., $\approx 1-2 \mathrm{~mm}$ as compared to the beam height of $20 \mathrm{~mm}$. Note that the uniaxial compliance as obtained in this paper [Eq. (18)] is related to the bending stresses with the axis of bending (i) normal to fiber direction and (ii) in the plane tangential to the annual rings. The so-obtained creep compliances are fitted with the expression for the extended Lomnitz law with the respective ramp compliance given in Eq. (8). For identification of the parameters $E, J_{\log }^{\mathrm{dev}}, \tau_{\log }$ and $\eta^{\mathrm{dev}}$, least-square fitting is employed, using the Levenberg-Marquardt algorithm, introducing the error

$$
\varepsilon=\sum_{i=1}^{n}\left[\widetilde{J}\left(t_{i},\left[E, J_{\log }^{\mathrm{dev}}, \tau_{\log }, \eta^{\mathrm{dev}}\right]\right)-J_{\text {test }}\left(t_{i}\right)\right]^{2} \rightarrow \min
$$

with data points given every $0.2 \mathrm{~s}$, fitting for the range $t>100 \mathrm{~s}$.

\section{Results and discussion}

\section{Uniqueness analysis of fitted material parameters}

The Lomnitz parameters obtained by least-square fitting were investigated for their uniqueness. The least-square error $\varepsilon$, normalized by its minimum value, is shown in Fig. 3 for a $80^{\circ} \mathrm{C}$ test. In Fig. 3a, the least-square error below a certain threshold value is plotted for a parameter range of $9000 \mathrm{MPa}<E<10500 \mathrm{MPa}, 10^{-3} \mathrm{~s}<\tau_{\log }<1 \mathrm{~s}$ and $3 \cdot 10^{-6} \mathrm{MPa}^{-1}<J_{\log }^{\text {dev }}<6 \cdot 10^{-6} \mathrm{MPa}^{-1}$, sampled with $200 \times 200 \times 200$ equally spaced points (linearly spaced with regard to $E$ and $J_{\log }^{\mathrm{dev}}$, logarithmically spaced with regard to $\tau_{\log }$ ), whereas the dashpot parameter $\eta^{\text {dev }}$ was set constant to the value found by least-square fitting. Obviously, the least-square error is not converging to a distinct minimum, but stretched over a large area in the three-parameter space (Fig. 3a) and in 
(a)

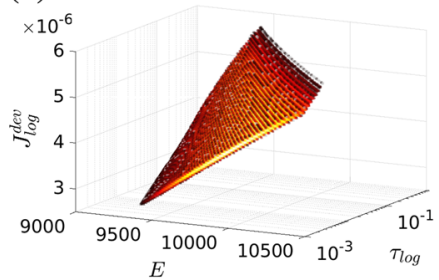

(b)

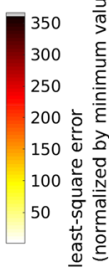

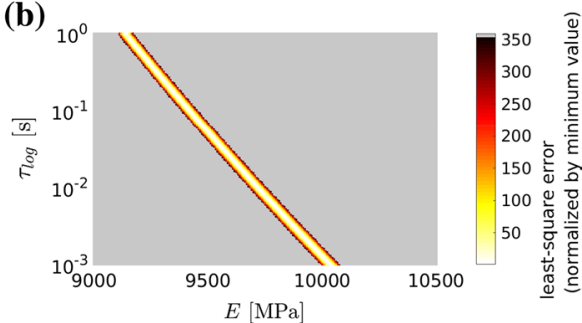

Fig. 3 Non-uniqueness of Lomnitz parameters: $\mathbf{a} \quad E, \quad J_{\log }^{\mathrm{dev}}$ and $\tau_{\log }$ and $\mathbf{b} \quad E$ and $\tau_{\log }$ for $J_{\log }^{\mathrm{dev}}=4.54 \times 10^{-6} \mathrm{MPa}^{-1}=$ const. The dashpot parameter $1 / \eta^{\mathrm{dev}}$ was set to $1 / \eta^{\mathrm{dev}}=3.36 \times 10^{-9} \mathrm{MPa}^{-1} \mathrm{~s}^{-1}=$ const

the $E-\tau_{\log }$ space (Fig. $3 \mathrm{~b}$ ); hence, the three parameters $E, J_{\log }^{\mathrm{dev}}$ and $\tau_{\log }$ are not independent. A similar dependency, most pronounced between parameters $E$ and $\tau_{\text {log }}$, has previously been observed when analyzing creep experiments for polyurethane foams (Pichler et al. 2018). The reasoning given in Pichler et al. (2018) was followed, i.e., $\tau_{\log }$ being probably too small to be reasonably obtained by standard creep (or relaxation) experiments. Hence, $\tau_{\log }$ is set to a constant value of $1 \mathrm{~s}$ what ensures uniqueness of the remaining three parameters as obtained below. A distinct minimum is found in both the three-dimensional parameter space (Fig. 4a) and the two-dimensional sections (Fig. $4 \mathrm{~b}-\mathrm{d}$ ) when setting $\tau_{\log }$ constant. The solution previously found by the Levenberg-Marquardt algorithm (denoted by black circles in Fig. 4b-d) corresponds to the color-coded minimum least-square error.

(a)
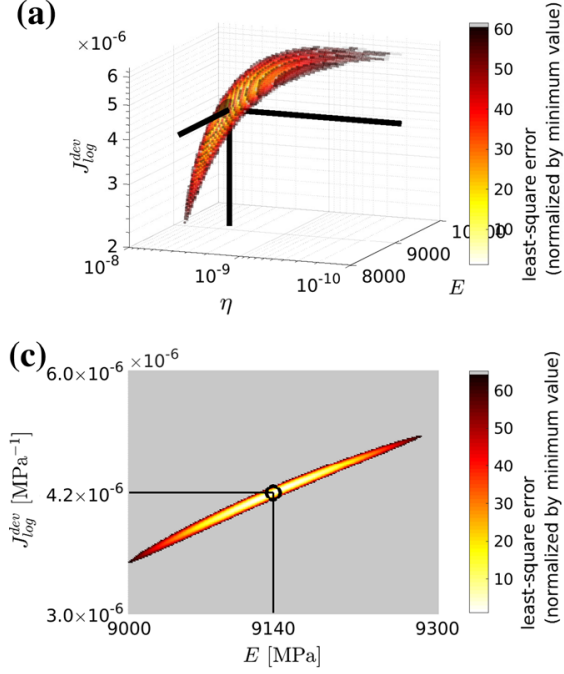

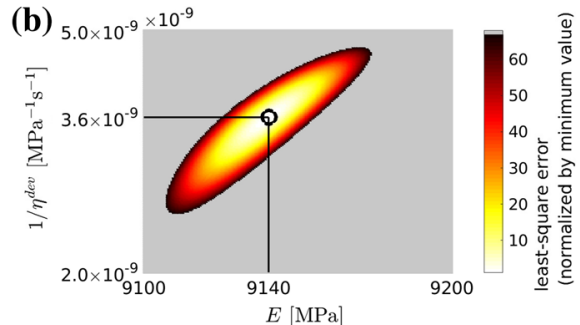

(d)

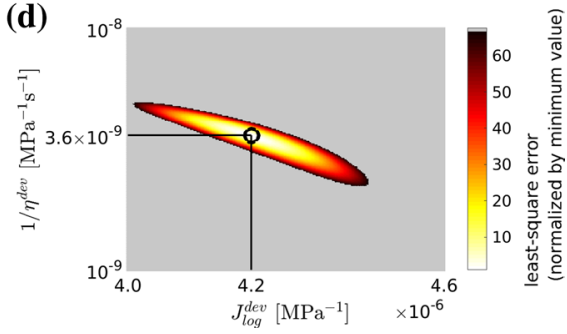

Fig. 4 Uniqueness of Lomnitz parameters: a $E, J_{\log }^{\text {dev }}$ and $1 / \eta^{\text {dev }}, \mathbf{b} E$ and $1 / \eta^{\text {dev }}$ for $J_{\log }^{\text {dev }}=4.54 \times 10^{-6} \mathrm{MPa}^{-1}=$ const., $\mathbf{c} E$ and $J_{\log }^{\text {dev }}$ for $1 / \eta^{\operatorname{dev}} \stackrel{\log }{=} 3.36 \times 10^{-9} \mathrm{MPa}^{-1} \mathrm{~s}^{-1}=$ const., $\mathbf{d} J_{\log }^{\text {dev }}$ and $1 / \eta^{\mathrm{dev}}$ for $E=9191 \mathrm{MPa}=$ const. The black circles denote the solution found by the Levenberg-Marquardt algorithm. The characteristic time was set $\tau_{\log }=1 \mathrm{~s}=$ const. 

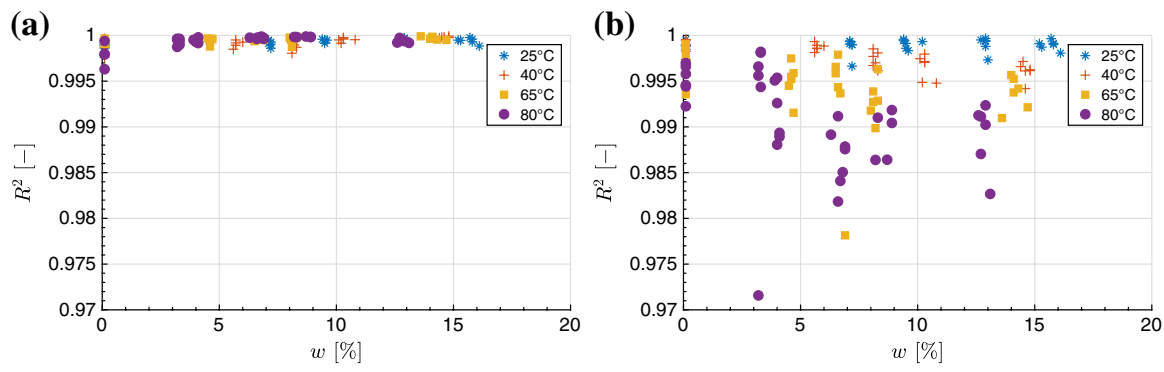

Fig. 5 Coefficient of determination $R^{2}$ for least-square fits of all creep tests, temperature levels color coded. The extended Lomnitz model (a) fits to the experimental data significantly better than the Lomnitz model without dashpot (b)
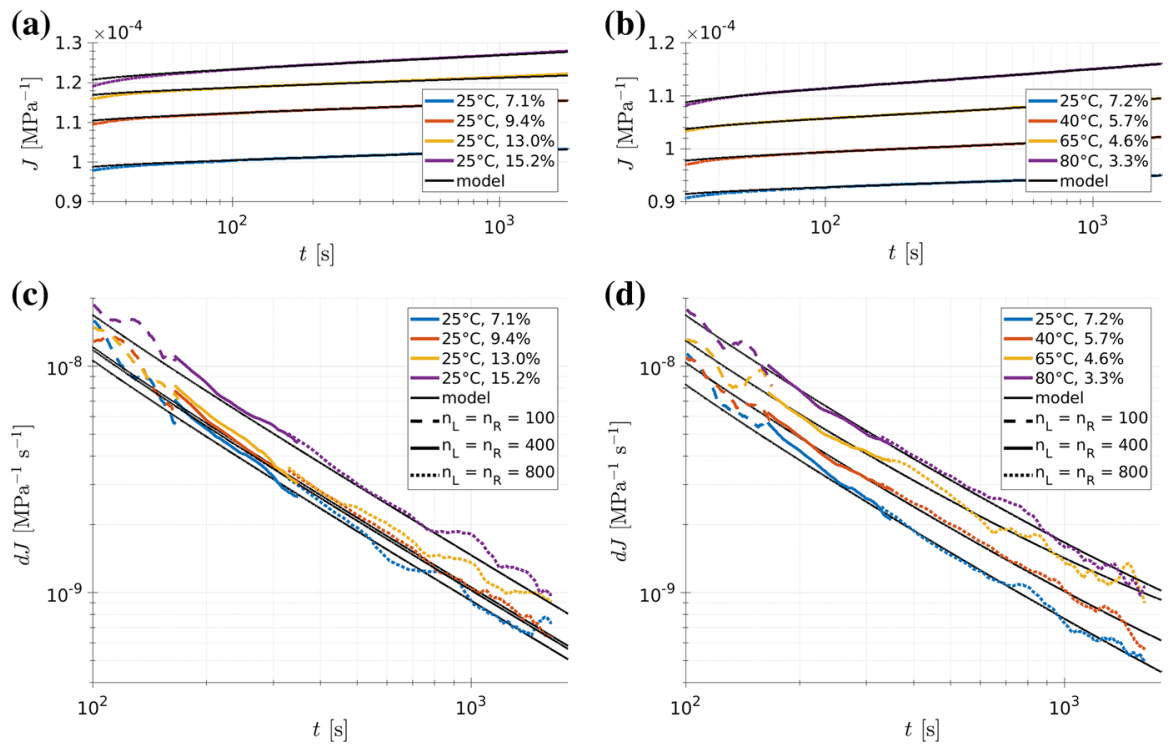

Fig. 6 Creep compliances derived from experiments with fitted ramp compliance $\widetilde{J}$ for varying temperature levels and moisture contents $(\mathbf{a}, \mathbf{b})$ along with creep compliance rates and respective ramp compliance derivatives $\tilde{\widetilde{J}}$ (c, d). Note that the moisture contents in the legends refer to single test results and, thus, are not matching with the average values given in Table 1

In order to assess the goodness of the employed least-square fitting procedure, the coefficient of determination $R^{2}$ is computed as

$$
R^{2}=1-\frac{\sum_{i}\left(J_{\text {test }}\left(t_{i}\right)-\widetilde{J}\left(t_{i}\right)\right)^{2}}{\sum_{i}\left(J_{\text {test }}\left(t_{i}\right)-\hat{J}_{\text {test }}\right)^{2}}
$$

with $\hat{J}$ as the average value of the test data points. Figure 5a shows the coefficients of determination when employing the extended Lomnitz model for parameter 
identification, whereas Fig. 5b depicts the coefficients of determination when using the Lomnitz model without a dashpot, for all tests, respectively. Whereas the Lomnitz model without additional dashpot exhibits a minimum $R^{2} \approx 0.972$, the extended Lomnitz model exhibits a minimum $R^{2} \approx 0.996$, hence, fits the test data significantly better.

Some of the obtained creep compliance functions are shown in Fig. 6a, b, their respective derivatives are given in Fig. $6 \mathrm{c}$, d. As the derivatives of the test data are obtained by numerical differentiation of the creep compliance functions and, hence, are rather noisy, a Savitzky-Golay filter (Savitzky and Golay 1964) (employing a second-order polynomial approximation) is applied to the experimental data, considering $100(t<160 \mathrm{~s}), 400(160<t<350 \mathrm{~s})$, and $800(350<t<1800 \mathrm{~s})$ left and right data points, respectively, for smoothing. The black lines represent the fitted curves of the extended Lomnitz model [Eq. (8)] and its derivative [Eq. (10)]. The extended Lommnitz models was fitted in the time domain (Fig. 6a, b) to each test, respectively, whereas the so-obtained Lomnitz parameters are also used for plotting the derivatives in Fig. 6c, d.
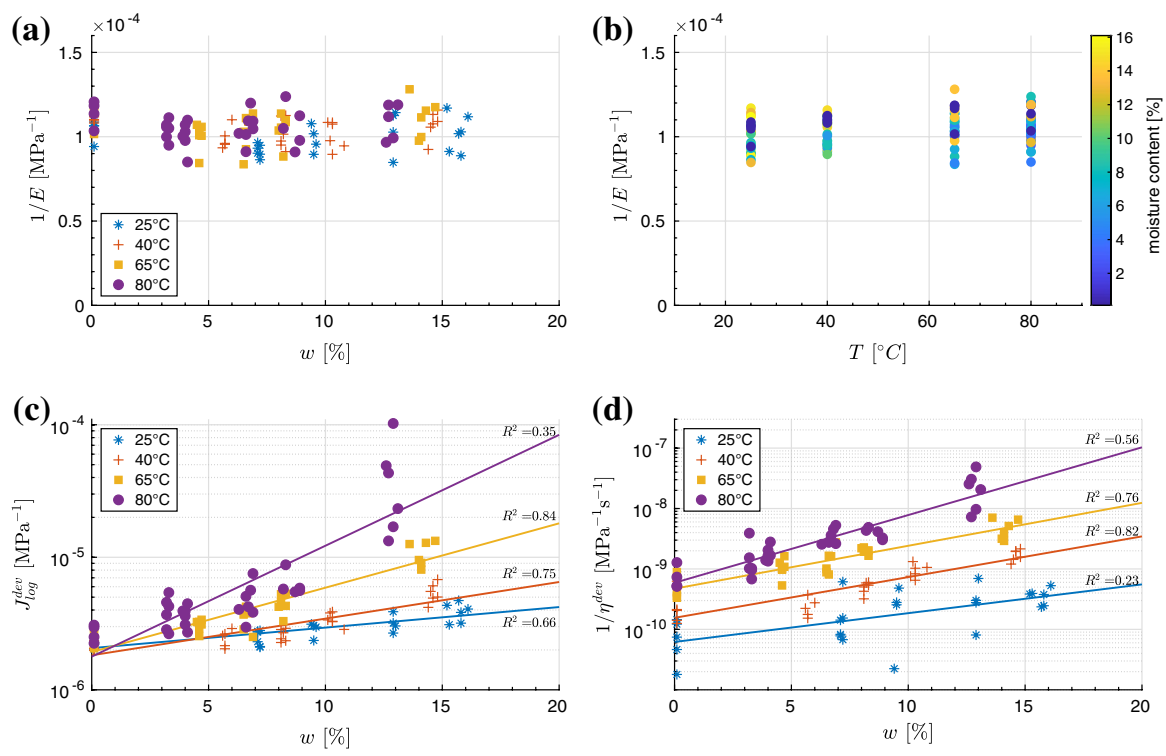

Fig. 7 Lomnitz parameters for varying moisture content for different temperature levels, including regression curves with corresponding coefficient of determination. Note: the coefficient of determination in $(\mathbf{c}, \mathbf{d})$ refers to the exponential fit of parameters determined in the "Uniqueness analysis of fitted material parameters" section. Although there is quite a scatter of data in $(\mathbf{c}, \mathbf{d})$, the obtained regression graphs are still suitable to interpret the general trend of the Lomnitz parameters with respect to temperature and moisture content 
Table 3 Lomnitz parameters obtained from least-square fitting of creep compliance data, mean value and standard deviations from six tests at each conditioning level

\begin{tabular}{lrlll}
\hline $\begin{array}{l}\text { Temperature } \\
\left({ }^{\circ} \mathrm{C}\right)\end{array}$ & \multicolumn{1}{l}{ rh $(\%)$} & $1 / E\left(\mathrm{MPa}^{-1}\right)$ & $J_{\log }^{\mathrm{dev}}\left(\mathrm{MPa}^{-1}\right)$ & $1 / \eta^{\mathrm{dev}}\left(\mathrm{MPa}^{-1} \mathrm{~s}^{-1}\right)$ \\
\hline 25 & $0.1 \pm 0.0$ & $1.04 \pm 0.05 \times 10^{-4}$ & $2.21 \pm 0.09 \times 10^{-6}$ & $8.12 \pm 4.69 \times 10^{-11}$ \\
& $7.1 \pm 0.1$ & $9.18 \pm 0.34 \times 10^{-5}$ & $2.47 \pm 0.30 \times 10^{-6}$ & $1.90 \pm 1.92 \times 10^{-10}$ \\
& $9.6 \pm 0.1$ & $9.87 \pm 0.68 \times 10^{-5}$ & $2.88 \pm 0.31 \times 10^{-6}$ & $2.60 \pm 1.63 \times 10^{-10}$ \\
& $12.9 \pm 0.0$ & $1.04 \pm 0.12 \times 10^{-4}$ & $3.20 \pm 0.44 \times 10^{-6}$ & $3.39 \pm 2.23 \times 10^{-10}$ \\
& $15.6 \pm 0.3$ & $1.02 \pm 0.10 \times 10^{-4}$ & $3.87 \pm 0.58 \times 10^{-6}$ & $3.61 \pm 0.99 \times 10^{-10}$ \\
& $0.1 \pm 0.0$ & $1.10 \pm 0.02 \times 10^{-4}$ & $2.14 \pm 0.14 \times 10^{-6}$ & $2.63 \pm 1.85 \times 10^{-10}$ \\
40 & $5.7 \pm 0.1$ & $9.91 \pm 0.59 \times 10^{-5}$ & $2.46 \pm 0.32 \times 10^{-6}$ & $2.40 \pm 0.79 \times 10^{-10}$ \\
& $8.2 \pm 0.1$ & $1.02 \pm 0.08 \times 10^{-4}$ & $2.58 \pm 0.25 \times 10^{-6}$ & $4.96 \pm 0.97 \times 10^{-10}$ \\
& $10.3 \pm 0.2$ & $1.01 \pm 0.07 \times 10^{-4}$ & $3.41 \pm 0.35 \times 10^{-6}$ & $9.42 \pm 2.23 \times 10^{-10}$ \\
& $14.6 \pm 0.1$ & $1.07 \pm 0.07 \times 10^{-4}$ & $5.42 \pm 0.84 \times 10^{-6}$ & $1.71 \pm 0.33 \times 10^{-9}$ \\
65 & $0.1 \pm 0.0$ & $1.14 \pm 0.07 \times 10^{-4}$ & $2.47 \pm 0.36 \times 10^{-6}$ & $4.98 \pm 2.28 \times 10^{-10}$ \\
& $4.6 \pm 0.1$ & $1.00 \pm 0.08 \times 10^{-4}$ & $3.08 \pm 0.28 \times 10^{-6}$ & $1.08 \pm 0.33 \times 10^{-9}$ \\
& $6.6 \pm 0.1$ & $1.02 \pm 0.11 \times 10^{-4}$ & $3.52 \pm 0.63 \times 10^{-6}$ & $1.46 \pm 0.67 \times 10^{-9}$ \\
& $8.2 \pm 0.1$ & $1.05 \pm 0.08 \times 10^{-4}$ & $4.51 \pm 0.69 \times 10^{-6}$ & $2.01 \pm 0.20 \times 10^{-9}$ \\
& $14.1 \pm 0.3$ & $1.12 \pm 0.10 \times 10^{-4}$ & $1.09 \pm 0.21 \times 10^{-5}$ & $4.80 \pm 1.60 \times 10^{-9}$ \\
& $0.1 \pm 0.0$ & $1.14 \pm 0.07 \times 10^{-4}$ & $2.70 \pm 0.34 \times 10^{-6}$ & $7.62 \pm 3.10 \times 10^{-10}$ \\
& $3.2 \pm 0.1$ & $1.04 \pm 0.05 \times 10^{-4}$ & $3.91 \pm 0.97 \times 10^{-6}$ & $1.52 \pm 1.10 \times 10^{-9}$ \\
& $4.0 \pm 0.1$ & $1.00 \pm 0.08 \times 10^{-4}$ & $3.63 \pm 0.58 \times 10^{-6}$ & $1.82 \pm 0.49 \times 10^{-9}$ \\
& $6.7 \pm 0.2$ & $1.05 \pm 0.09 \times 10^{-4}$ & $4.30 \pm 0.86 \times 10^{-6}$ & $3.15 \pm 0.72 \times 10^{-9}$ \\
& $8.3 \pm 0.7$ & $1.06 \pm 0.10 \times 10^{-4}$ & $6.50 \pm 1.23 \times 10^{-6}$ & $4.16 \pm 0.80 \times 10^{-9}$ \\
& $12.8 \pm 0.2$ & $1.26 \pm 0.38 \times 10^{-4}$ & $4.14 \pm 3.02 \times 10^{-5}$ & $2.37 \pm 1.39 \times 10^{-8}$ \\
\hline & & & &
\end{tabular}

\section{Discussion}

In Fig. 7, the Lomnitz parameters obtained by least-square fitting are depicted as functions of the moisture content and temperature; Table 3 shows the mean values and standard deviations of all Lomnitz parameters with regard to the conditioning classes defined in Table 1. The results shown in Fig. 7c, d were least-square fitted by exponential functions (appearing as linear functions since the ordinate is plotted in logarithmic scaling), emphasizing the trend in Lomnitz parameters.

Figure $7 \mathrm{a}, \mathrm{b}$ shows that the increase in the compliance $1 / E$ with moisture content and temperature, respectively, is almost negligible when compared to the other parameters plotted in Fig. $7 \mathrm{c}$, d, i.e., $J_{\log }^{\mathrm{dev}}$ and $1 / \eta^{\mathrm{dev}}$. Figure $7 \mathrm{c}$, d shows the dependency of the Lomnitz parameters $J_{\log }^{\operatorname{dev}}$ and $1 / \eta^{\text {dev }}$ on the moisture content. Both parameters $J_{\log }^{\mathrm{dev}}$ and $1 / \eta^{\mathrm{dev}}$ increase with increasing temperature and moisture content. The dependency of creep parameters $J_{\log }^{\text {dev }}$ and $1 / \eta^{\text {dev }}$ is consistent with previous results, showing that increasing the temperature and moisture content leads to a more pronounced creep behavior (c.f. Jiang et al. 2009; Hering and Niemz 2012). 

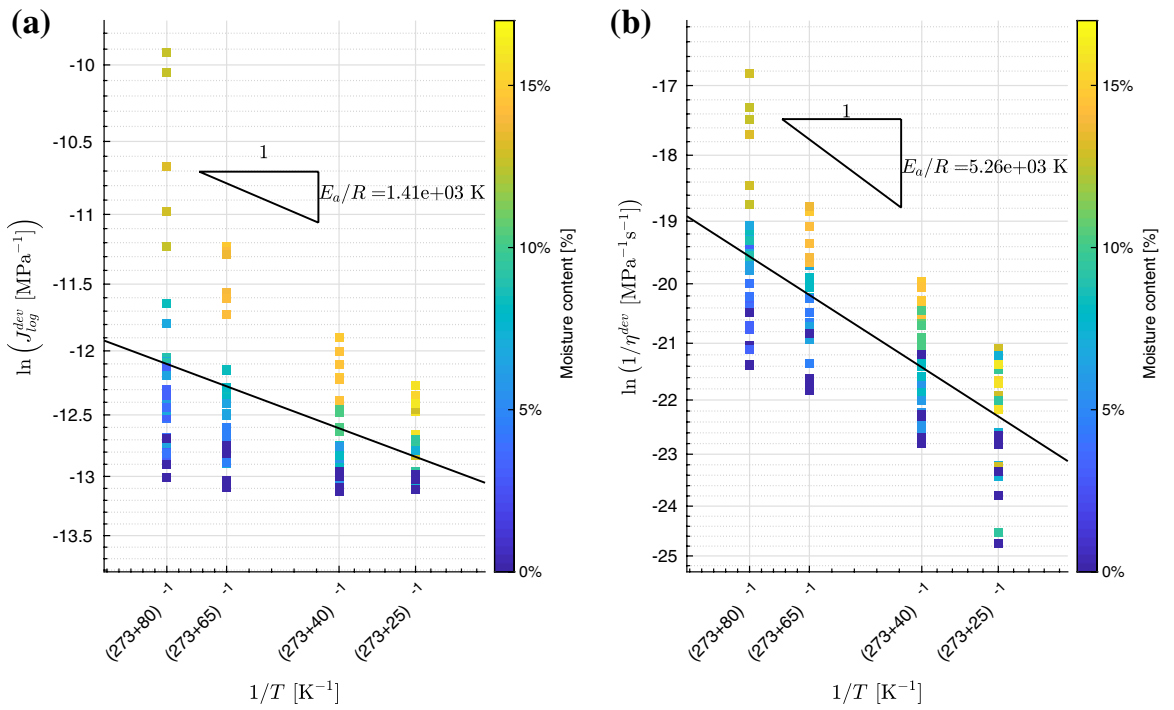

Fig. 8 Arrhenius plot for back-calculated Lomnitz parameters along with slope representing the activation energy, moisture content visualized by color code

Figure 8 shows an Arrhenius plot for the Lomnitz parameters $J_{\log }^{\mathrm{dev}}$ and $1 / \eta^{\mathrm{dev}}$, with the moisture content visualized by color coding. The black lines represent fitted Arrhenius equations to the test data, reading (Navi and Stanzl-Tschegg 2009)

$$
\text { - }=A \exp \left(-\frac{E_{a}}{R T}\right)
$$

with $\bullet$ as $J_{\log }^{\mathrm{dev}}$ or $1 / \eta^{\mathrm{dev}}$, respectively, $A$ as a pre-exponential factor and $E_{a}\left(\mathrm{~J} \mathrm{~mol}^{-1}\right)$ as the activation energy of the creep process, $R=8.314 \mathrm{~J} \mathrm{~mol}^{-1} \mathrm{~K}^{-1}$ as the gas constant and $T(\mathrm{~K})$ as the absolute temperature. By taking the logarithm, Eq. (21) is written as

$$
\ln (\bullet)=-\frac{E_{a}}{R} \frac{1}{T}+\ln (A)
$$

i.e., linear in terms of the independent variable $1 / T$; hence, a linear function is fitted to the logarithmic Lomnitz-type creep parameters $\ln \left(J_{\log }^{\mathrm{dev}}\right)$ and $\ln \left(1 / \eta^{\mathrm{dev}}\right)$, respectively, vs. $1 / T$ (Fig. 8). The activation energy represents the slope in the Arrhenius diagram and is given by $E_{a}=11.8 \mathrm{~kJ} / \mathrm{mol}$ and $E_{a}=43.7 \mathrm{~kJ} / \mathrm{mol}$ for $J_{\log }^{\mathrm{dev}}$ and $1 / \eta^{\mathrm{dev}}$, respectively. As all materials exhibit, in general, different activation energies (see e.g., Lomellini (1992) and Eckstein et al. (1997) for activation energies of polymers), the difference in activation energies for $J_{\log }^{\text {dev }}$ and $1 / \eta^{\text {dev }}$ could indicate that different material phases are involved in the creep mechanism of wood. According to Kelley et al. (1987), amorphous lignin and hemicellulose exhibit a different viscoelastic behavior, both being strongly dependent on heat and moisture content. One 
Table 4 Lomnitz parameters obtained from relaxation tests at four different temperature levels

\begin{tabular}{lllll}
\hline $\begin{array}{l}\text { Tem- } \\
\text { perature } \\
\left({ }^{\circ} \mathrm{C}\right)\end{array}$ & $\mathrm{rh}(\%)$ & $1 / E\left(\mathrm{MPa}^{-1}\right)$ & $J_{\log }^{\mathrm{dev}} \mathrm{MPa}^{-1}$ & $1 / \eta^{\mathrm{dev}}\left(\mathrm{MPa}^{-1} \mathrm{~s}^{-1}\right)$ \\
\hline 25 & 14.6 & $1.04 \times 10^{-4}$ & $4.80 \times 10^{-6}$ & $5.00 \times 10^{-10}$ \\
40 & 14.8 & $1.21 \times 10^{-4}$ & $5.90 \times 10^{-6}$ & $1.11 \times 10^{-9}$ \\
65 & 14.8 & $1.22 \times 10^{-4}$ & $1.55 \times 10^{-5}$ & $1.82 \times 10^{-8}$ \\
80 & 12.3 & $1.50 \times 10^{-4}$ & $2.00 \times 10^{-5}$ & $2.00 \times 10^{-8}$ \\
\hline
\end{tabular}
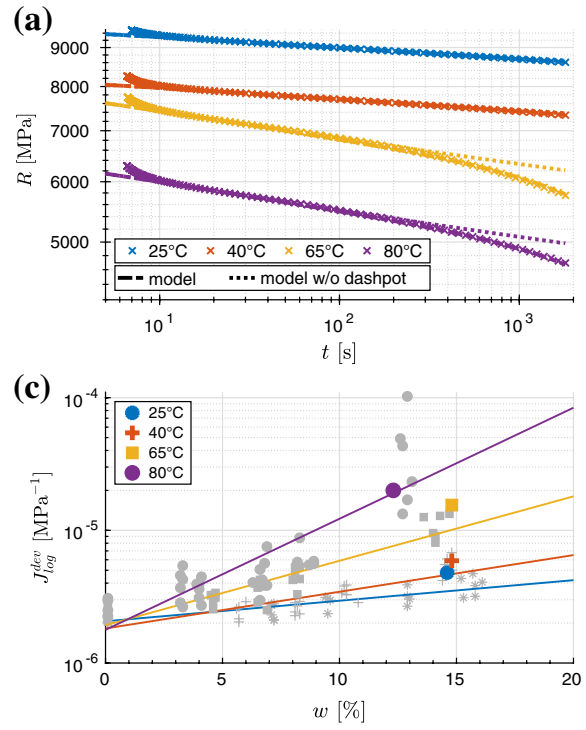
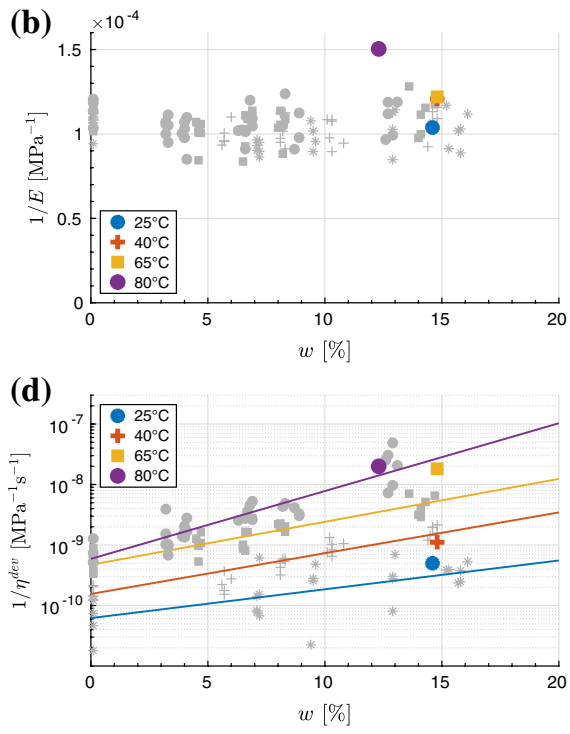

Fig. 9 a Relaxation test data with back-calculated Lomnitz models, with and without additional dashpot. Note that the influence of the dashpot within the extended Lomnitz model, compared to the Lomnitz model without dashpot, increases with increasing temperature, which can also be seen in (d) and is consistent with the values given in Table 4. b-d Lomnitz parameters (in gray) and regression lines obtained from creep tests, c.f. Fig. 7. The colored dots originate from the relaxation tests and compare well to the parameters obtained from creep tests

may explain the different activation energies of the Lomnitz parameters in the macroscopic wood specimens with the differences in the viscoelastic behavior of those polymers at the microscale.

Relaxation tests ("Experimental work" section) are used for verification of the proposed viscoelastic model. As shown in Fig. 9a, the begin of the relaxation phase is influenced by the finite displacement application time, however, for $t>\approx 20 \mathrm{~s}$ the 
extended Lomnitz model fits the relaxation curves rather good. The obtained Lomnitz parameters are summarized in Table 4 and depicted in Fig. 9. Those parameters were not obtained by least-square fitting, since the relaxation function Eqs. (13)-(16) can only be expressed as a Volterra integral equation, which is solved numerically using the algorithm outlined in Press et al. (1992); hence, the Lomnitz parameters were determined visually (Fig. 9a). The so-obtained Lomnitz parameters $1 / E, J_{\log }^{\mathrm{dev}}$ and $1 / \eta^{\mathrm{dev}}$ are shown in Fig. 9b-d as colored dots; the gray dots and the regression lines were previously determined from the creep tests (Fig. 7). The Lomnitz parameters determined from relaxation tests agree well with the parameters obtained from creep tests; hence, the relaxation experiments may be regarded as a verification of Lomnitz-type behavior identified from creep tests.

\section{Conclusion}

Based on half-hour long creep tests on clear spruce specimens performed at constant moisture content to deactivate potential mechano-sorptive creep phenomena, the viscoelastic behavior was found to obey a Lomnitz model extended by a Newtonian dashpot. Thereby, the influence of temperature and moisture content was taken into account. The extended Lomnitz model was shown to provide a good fit to the test data at all investigated levels of temperature and moisture content with a limited number of only three model parameters. The respective model parameters $E, J_{\log }^{\mathrm{dev}}$ and $\eta^{\text {dev }}$ were back-calculated using a least-square fitting algorithm, whereas the characteristic time $\tau_{\log }$ was set to a fixed value of $\tau_{\log }=1 \mathrm{~s}$. The uniqueness of parameters was shown by visualization of the least-square error in the three-parameter space. While the dependence of the elastic compliance $1 / E$ on temperature and moisture content was shown to be comparatively small, a pronounced dependency of the Lomnitz parameters $J_{\log }^{\mathrm{dev}}$ and $1 / \eta^{\mathrm{dev}}$ on moisture content and temperature was observed, both increasing with increasing temperature and moisture content. Arrhenius plots gave access to a distinctively different temperature dependency of Lomnitz parameters $J_{\log }^{\mathrm{dev}}$ and $1 / \eta^{\mathrm{dev}}$. That may indicate the presence of at least two different creep processes at the microscale of the material, which could be caused by the different viscoelastic behavior of the amorphous wood constituents hemicellulose and lignin, respectively. In order to confirm the applicability of the Lomnitz law as regards time-dependent behavior of wood, the obtained material parameters were validated by relaxation tests.

Acknowledgments Open access funding provided by University of Innsbruck and Medical University of Innsbruck.

\section{Compliance with ethical standards}

Conflict of interest On behalf of all authors, the corresponding author states that there is no conflict of interest. 
Open Access This article is distributed under the terms of the Creative Commons Attribution 4.0 International License (http://creativecommons.org/licenses/by/4.0/), which permits unrestricted use, distribution, and reproduction in any medium, provided you give appropriate credit to the original author(s) and the source, provide a link to the Creative Commons license, and indicate if changes were made.

\section{References}

Acker P, Ulm FJ (2001) Creep and shrinkage of concrete: physical origins and practical measurements. Nucl Eng Des 203(2-3):143-158. https://doi.org/10.1016/S0029-5493(00)00304-6

Altoubat SA, Lange DA (2002) The Pickett effect at early age and experiment separating its mechanisms in tension. Mater Struct 35(4):211-218. https://doi.org/10.1007/BF02533082

Armstrong L, Kingston R (1960) The effect of moisture changes on creep in wood. Nature 185:862-863. https://doi.org/10.1038/185862c0

Austrian Standards International (A.S.I.) (2003) ÖNORM B 3012: wood species—characteristic values to terms and symbols of ÖNORM EN 13556

Bach L, Pentoney RE (1968) Nonlinear mechanical behavior of wood. For Prod J 18(3):60-66

Bader TK, Hofstetter K, Hellmich C, Eberhardsteiner J (2011) The poroelastic role of water in cell walls of the hierarchical composite "softwood". Acta Mech 217(1-2):75-100. https://doi.org/10.1007/ s00707-010-0368-8

Bažant ZP (1985) Constitutive equation of wood at variable humidity and temperature. Wood Sci Technol 19(2):159-177. https://doi.org/10.1007/BF00353077

Bažant ZP, Meiri S (1985) Measurements of compression creep of wood at humidity changes. Wood Sci Technol 19(2):179-182. https://doi.org/10.1007/BF00353079

Bažant Z, Prasannan S (1989) Solidification theory for concrete creep. I: formulation. J Eng Mech 115(8):1691-1703. https://doi.org/10.1061/(ASCE)0733-9399(1989)115:8(1691)

Bažant ZP, Xi Y (1993) New test method to separate microcracking from drying creep: curvature creep at equal bending moments and various axial forces. In: Bažant ZP, Carol I (eds) RILEM Proceedings. Chapman \& Hall, Barcelona, pp 77-82

Bažant ZP, Hauggaard AB, Baweja S, Ulm FJ (1997) Microprestress-solidification theory for concrete creep. I: aging and drying effects. J Eng Mech 123(11):1188-1194. https://doi.org/10.1061/ (ASCE)0733-9399(1997)123:11(1188)

Beijer JGJ, Spoormaker JL (2002) Solution strategies for FEM analysis with nonlinear viscoelastic polymers. Comput Struct 80(14-15):1213-1229. https://doi.org/10.1016/S0045-7949(02)00089-5

Bekhta P, Niemz P (2003) Effect of high temperature on physical and mechanical properties of spruce wood. Holzforschung 57(5):539-546. https://doi.org/10.1515/HF.2003.080

Bodig J, Jayne BA (1982) Mechanics of wood and wood composites. Van Nostrand Reinhold, New York

Brinson HF, Brinson LC (2008) Polymer engineering science and viscoelasticity: an introduction. Springer, New York. https://doi.org/10.1007/978-1-4899-7485-3

Clouser WS (1959) Creep of small wood beams under constant bending load. Tech. rep, Forest Products Laboratory, Madison, WI

Eckstein A, Friedrich C, Lobbrecht A, Spitz R, Mülhaupt R (1997) Comparison of the viscoelastic properties of syndio- and isotactic polypropylenes. Acta Polym 48(1-2):41-46. https://doi.org/10.1002/ actp.1997.010480107

Eitelberger J, Bader TK, De Borst K, Jäger A (2012) Multiscale prediction of viscoelastic properties of softwood under constant climatic conditions. Comput Mater Sci 55:303-312. https://doi. org/10.1016/j.commatsci.2011.11.033

Flügge W (1975) Viscoelasticity, 2nd edn. Springer, Berlin. https://doi.org/10.1007/978-3-662-02276-4

Greenspan L (1977) Humidity fixed points of binary saturated aqueous solutions. J Res Nat Bur Stand 81A(1):89-96. https://doi.org/10.6028/jres.081A.011

Haque MN, Langrish TA, Keep LB, Keey RB (2000) Model fitting for visco-elastic creep of Pinus radiata during kiln drying. Wood Sci Technol 34(5):447-457. https://doi.org/10.1007/s002260000 058

Hering S, Niemz P (2012) Moisture-dependent, viscoelastic creep of European beech wood in longitudinal direction. Eur J Wood Prod 70(5):667-670. https://doi.org/10.1007/s00107-012-0600-4 
Hofer U, Luger M, Traxl R, Lackner R (2018) Closed-form expressions for effective viscoelastic properties of fiber-reinforced composites considering fractional matrix behavior. Mech Mater 127:14-25. https://doi.org/10.1016/j.mechmat.2018.08.005

Hofstetter K, Hellmich C, Eberhardsteiner J (2005) Development and experimental validation of a continuum micromechanics model for the elasticity of wood. Eur J Mech A Solids 24(6):1030-1053. https://doi.org/10.1016/j.euromechsol.2005.05.006

Holzer SM, Loferski JR, Dillard DA (1989) A review of creep in wood: concepts relevant to develop long-term behavior predictions for wood structures. Wood Fiber Sci 21(4):376-392

Hoyle RJ, Griffith MC, Itani RY (1985) Primary creep in Douglas-fir beams of commercial size and quality. Wood Fiber Sci 17(3):300-314

Hunt DG (1999) A unified approach to creep of wood. Proc R Soc Lond A 455(1991):4077-4095. https:// doi.org/10.1098/rspa.1999.0491

Hunt DG (2004) The prediction of long-time viscoelastic creep from short-time data. Wood Sci Technol 38(7):479-492. https://doi.org/10.1007/s00226-004-0244-6

Hunt DG, Shelton CF (1988) Longitudinal moisture-shrinkage coefficients of softwood at the mechanosorptive creep limit. Wood Sci Technol 22(3):199-210. https://doi.org/10.1007/BF00386014

Idesman A, Niekamp R, Stein E (2001) Finite elements in space and time for generalized viscoelastic maxwell model. Comput Mech 27(1):49-60. https://doi.org/10.1007/s004660000213

Jiang J, Lu J, Huang R, Li X (2009) Effects of time and temperature on the viscoelastic properties of Chinese fir wood. Drying Technol 27(11):1229-1234. https://doi.org/10.1080/07373930903266726

Kelley SS, Rials TG, Glasser WG (1987) Relaxation behaviour of the amorphous components of wood. J Mater Sci 22(2):617-624. https://doi.org/10.1007/BF01160778

King EG (1961) Time-dependent strain behavior of wood. For Prod J 11(3):156-165

Lomellini P (1992) Williams-Landel-Ferry versus Arrhenius behaviour: polystyrene melt viscoelasticity revised. Polymer 33(23):4983-4989. https://doi.org/10.1016/0032-3861(92)90049-3

Lomnitz C (1956) Creep measurements in igneous rocks. J Geol 65(5):473-479. https://doi. org/10.1086/626379

Lomnitz C (1957) Linear dissipation in solids. J Appl Phys 28(2):201-205. https://doi. org/10.1063/1.1722707

Lomnitz C (1962) Application of the logarithmic creep law to stress wave attenuation in the solid earth. J Geophys Res 67(1):365-368. https://doi.org/10.1029/JZ067i001p00365

Mainardi F, Spada G (2012) On the viscoelastic characterization of the Jeffreys-Lomnitz law of creep. Rheol Acta 51(9):783-791. https://doi.org/10.1007/s00397-012-0634-X

Mohager S, Toratti T (1992) Long term bending creep of wood in cyclic relative humidity. Wood Sci Technol 27(1):49-59. https://doi.org/10.1007/BF00203409

Morlier P (1994) Creep in timber structures. CRC Press, London. https://doi.org/10.1201/9781482294 750

Mukudai J (1983) Evaluation on non-linear viscoelastic bending deflection of wood. Wood Sci Technol 17(1):39-54. https://doi.org/10.1007/BF00351831

Mukudai J, Yata S (1986) Modeling and simulation of viscoelastic behavior (tensile strain) of wood under moisture change. Wood Sci Technol 20(4):335-348. https://doi.org/10.1007/BF00351586

Nabarro FRN (2001a) Creep mechanisms in crystalline solids. In: Encyclopedia of materials: science and technology. Elsevier, pp 1788-1795

Nabarro FRN (2001b) The time constant of logarithmic creep and relaxation. Mater Sci Eng A 309310:227-228. https://doi.org/10.1016/S0921-5093(00)01692-0

Nakano T (2008) Analysis of cell wall swelling on the basis of a cylindrical model. Holzforschung 62(3):352-356. https://doi.org/10.1515/HF.2008.042

Navi P, Stanzl-Tschegg S (2009) Micromechanics of creep and relaxation of wood. A review. Holzforschung 63(2):186-195. https://doi.org/10.1515/HF.2009.013

Ozyhar T, Hering S, Niemz P (2013) Viscoelastic characterization of wood: time dependence of the orthotropic compliance in tension and compression. J Rheol 57(2):699-717. https://doi. org/10.1122/1.4790170

Pandey V, Holm S (2016) Linking the fractional derivative and the Lomnitz creep law to non-Newtonian time-varying viscosity. Phys Rev E 94(3):032606. https://doi.org/10.1103/PhysRevE.94.032606

Pichler C, Lackner R (2009) Identification of logarithmic-type creep of calcium-silicate-hydrates by means of nanoindentation. Strain 45(1):17-25. https://doi.org/10.1111/j.1475-1305.2008.00429.x 
Pichler C, Lackner R, Mang HA (2008) Multiscale model for creep of shotcrete-from logarithmic-type viscous behavior of $\mathrm{CSH}$ at the $\mu \mathrm{m}$-scale to macroscopic tunnel analysis. J Adv Concr Technol 6(1):91-110. https://doi.org/10.3151/jact.6.91

Pichler C, Maier M, Lackner R (2018) Viscoelastic response of closed-cell polyurethane foams from half hour-long creep tests: identification of Lomnitz behavior. J Eng Mater Technol 141(2):021001. https ://doi.org/10.1115/1.4040831

Pickett G (1942) The effect of change in moisture content on the creep of concrete under a sustained load. ACI J Proc 38:333-356. https://doi.org/10.14359/8607

Pipkin AC (1986) Lectures on viscoelasticity theory. Springer, New York

Placet V, Passard J, Perre P (2008) Viscoelastic properties of wood across the grain measured under water-saturated conditions up to $135^{\circ}$ C: evidence of thermal degradation. J Mater Sci 43(9):3210 3217. https://doi.org/10.1007/s10853-008-2546-9

Press WH, Teukolsky SA, Vetterling WT, Flannery BP (1992) Numerical recipes in Fortran 77-the art of scientific computing, 2nd edn. Cambridge University Press, Cambridge

Ranta-Maunus A (1975) The viscoelasticity of wood at varying moisture content. Wood Sci Technol 9(3):189-205. https://doi.org/10.1007/BF00364637

Savitzky A, Golay MJ (1964) Smoothing and differentiation of data by simplified least squares procedures. Anal Chem 36(8):1627-1639. https://doi.org/10.1021/ac60214a047

Schänzlin J (2010) Modeling the long-term behavior of structural timber for typical serviceclass-II-conditions in South-West Germany. Habilitation thesis, University of Stuttgart. https://doi.org/10.18419 lopus-348

Schniewind AP, Barrett JD (1972) Wood as a linear orthotropic viscoelastic material. Wood Sci Technol 6:43-57. https://doi.org/10.1007/BF00351807

Schroeder HA (1972) Shrinking and swelling differences between hardwoods and softwoods. Wood Fiber Sci 4(1):20-25

Singh SJ, Rosenman M (1974) Quasi-static deformation of a viscoelastic half-space by a displacement dislocation. Phys Earth Planet Inter 8(1):87-101. https://doi.org/10.1016/0031-9201(74)90114-9

Stamm AJ (1935) Shrinking and swelling of wood. Ind Eng Chem 27(4):401-406. https://doi. org/10.1021/ie50304a011

Stamm AJ (1956) Thermal degradation of wood and cellulose. Ind Eng Chem 48(3):413-417. https://doi. org/10.1021/ie51398a022

Ward IM, Sweeney J (2012) Mechanical properties of solid polymers, 3rd edn. Wiley, Chichester. https:// doi.org/10.1002/9781119967125

Publisher's Note Springer Nature remains neutral with regard to jurisdictional claims in published maps and institutional affiliations 